Check for updates

1 Institute of Health and Wellbeing, University of Glasgow, Glasgow, UK

2 Knowledge and Evaluation Research Unit, Mayo Clinic, Rochester MN, USA

3 The Patient Revolution, Inc., Rochester, MN, USA

4 London School of Hygiene and Tropical Medicine, London, UK

5 NIHR North London Applied Research Collaborative, London, UK

Cite this as: BMJ 2021;375:n2909 http://dx.doi.org/10.1136/bmj.n2909 Published: 25 November 2021

\section{Digital transformation could increase the burden of treatment on patients}

\author{
Frances S Mair, ${ }^{1}$ Victor M Montori, ${ }^{2,3}$ Carl R May ${ }^{4,5}$
}

The burden of treatment refers to the demands that healthcare professionals and systems place on patients and their support community, and the implications this has on their quality of life. ${ }^{1}$ It is an important barometer of quality of care. It includes the work involved in self-management of care such as: gaining an understanding of chronic conditions, their management and implications; engaging with health and social care professionals, family, and friends to access care and treatments; putting self-management recommendations into practice; taking multiple medications; enduring side effects; attending appointments and investigations; enacting lifestyle and behaviour changes; self-monitoring activities and reconfiguring treatments to make them workable; and navigating complex care systems.

Many factors have been recognised as increasing the burden of treatment such as multimorbidity and associated polypharmacy; inadequate provision of information; the administrative burden associated with managing a chronic illness, such as dealing with poorly coordinated healthcare; and inadequate communication.

People vary in their capacity to cope with any given level of burden of treatment depending on factors ranging from the individual (cognitive abilities, health literacy, physical condition) to wider issues such as their financial circumstances, environment, and the strength of their support community. ${ }^{2}$

Digital transformation is viewed as a potential solution to the challenges facing healthcare systems internationally, but if it results in more workload shifting to patients and their support communities, it could increase rather than lessen the burden of treatment. The NHS Long Term Plan aims to provide a "digital first" option within 10 years. ${ }^{3}$ The current chancellor of the exchequer says that he plans to spend "billions on digitising the NHS." " Digital health provides opportunities and challenges, particularly to ensure that the move to digital health does not widen health inequalities. Scant consideration has been given to how, and to what extent, digital health ameliorates or contributes to the burden of treatment experienced by patients and their supporters.

Digital health has the potential to reduce the burden of treatment for some. The ability to tele-consult (via telephone or video) can reduce the number of visits to health professionals. Attending clinic or hospital appointments is time consuming, can be stressful, and may entail considerable expense and effort. The distance a person must travel, availability and affordability of transportation, building accessibility, and whether the appointment requires the person to negotiate time off work or childcare, can all influence the perceived burden of treatment associated with attending appointments. Reducing these visits through teleconsulting can lessen the burden of treatment for those with busy home or work lives, for those for whom accessing transportation is difficult or expensive, or those with disabilities (visible or invisible). However, teleconsulting via video requires access to fast broadband and wi-fi in the home and assumes that people can use these systems. People who are homeless, or those from less affluent groups will not have access to these resources and may be seriously disadvantaged. They will find the burden of treatment exacerbated by a move to digital or remote care. Similarly, those who do not speak English, or who have cognitive or other sensory impairments may also find the move to remote care increases the burden of treatment as they are unable to access digital services unaided.

The promotion of home telemonitoring systems for people with chronic conditions such as hypertension or asthma may lessen the treatment burden for some as it may decrease face-to-face appointments and may enhance people's knowledge and understanding of their condition and an individual's sense of control. However, some of the time saved will be substituted with additional self-monitoring work. Whether it succeeds in lessening treatment burden will depend on the individual's personal circumstances as outlined above. Furthermore, a person with multiple chronic conditions who has multiple separate home telemonitoring systems to operate may find the self-monitoring workload overwhelming and unmanageable.

Evidence-based apps for people to promote weight loss or help manage low back pain or diabetes may lessen the burden of treatment if users find the apps to be useful, usable, and desirable. When, on the other hand, apps are poorly integrated into plans of care, offer misinformation or poor-quality advice, or are demanding to use, they may increase the burden of treatment. This may also be the case when users are overwhelmed by demands (made by life or healthcare) on their time which they must face alone because of the move to remote care. In either case, patients may feel abandoned by healthcare that by offering digital "solutions" is not just unresponsive, but also burdensome.

The move to remote digital healthcare can reduce the burden of treatment to the extent that these services are purposefully designed primarily to reduce the demands on time, energy, and attention placed on the patient, and only secondarily to reduce costs or improve operational efficiency. Devices, applications, workflows, systems, and practices must be designed to avoid exceeding the capacity of those less digitally able-because of financial, language, literacy, 
cognitive, physical, and social support limitations. In addition, an overemphasis on disease focused digital health solutions will be inappropriate for the growing population that lives with multimorbidity (two or more chronic health conditions). Digital and remote healthcare not designed with these factors in mind will render patients vulnerable, not only to digital inequalities, but to loss of wellbeing due to their contribution to an already unsustainable burden of treatment.

Competing interests: FM: While I have no personal financial conflicts of interest I have potential organisational conflicts of interest as I have been Principle or Co-Investigator on external funding from the Medical Research Council; Engineering and Physical Sciences Research Council; Economic and Social Research Council; EU Horizon 2020; NIHR/UKRI; Chief Scientist Office Scotland; Innovate UK; British Heart Foundation; Dunhill Trust; Wellcome Trust; and Marie Curie to undertake research on multimorbidity, treatment burden and digital health, where my University has received payments. I also promote the idea of "minimally disruptive medicine" (but do not derive any income from this work). The selfBACK app for low back pain developed through a Horizon 2020 project is now being commercialised and my University may gain financially from this, however I will not derive any income from this. VM: I have collaborated in the development of measures of treatment burden and chair the board of the Patient Revolution, a nonprofit that advocates for careful and kind care, including minimally disruptive medicine. I have derived and will derive no income from these activities. CM: none declared.

1 Mair FS, May CR. Thinking about the burden of treatment. BM/2014;349:g6680 doi: 10.1136/bmj.g6680 pmid: 25385748

2 Shippee ND, Shah ND, May CR, Mair FS, Montori VM. Cumulative complexity: a functional, patient-centered model of patient complexity can improve research and practice. J Clin Epidemiol 2012;65:1041-51. doi: 10.1016/j.jlinepi.2012.05.005 pmid: 22910536

3 NHS. The NHS long term plan. 2019. https://www.longtermplan.nhs.uk/

4 Rishi Sunak to spend billions to digitise NHS. The Telegraph 23 October 21. Accessed 24 October 21. https://www.telegraph.co.uk/politics/2021/10/23/rishi-sunak-spend-billions-digitise-nhs/ 